Revue d'histoire de l'Amérique française

ZWB REVUE D.HISTOIRE DE L'AMÉRIQUE FRANÇAISE

\title{
Les débuts de l'enseignement de l'histoire et de la géographie au Petit Séminaire de Québec (1765-1830)
}

\section{Pierre Savard}

Volume 15, numéro 4, mars 1962

URI : https://id.erudit.org/iderudit/302153ar

DOI : https://doi.org/10.7202/302153ar

Aller au sommaire du numéro

Éditeur(s)

Institut d'histoire de l'Amérique française

ISSN

0035-2357 (imprimé)

1492-1383 (numérique)

Découvrir la revue

Citer cet article

Savard, P. (1962). Les débuts de l'enseignement de l'histoire et de la géographie au Petit Séminaire de Québec (1765-1830). Revue d'histoire de l'Amérique française, 15(4), 509-525. https://doi.org/10.7202/302153ar d'utilisation que vous pouvez consulter en ligne. 


\title{
LES DÉBUTS DE L'ENSEIGNEMENT DE L'HISTOIRE ET DE LA GÉOGRAPHIE AU PETIT SÉMINAIRE DE QUÉBEC *
}

\author{
$(1765-1830)$
}

I

En 1759, au plus fort de la Guerre de la Conquête, le Collège de Québec ferme ses portes. En 1762 les Jésuites sont supprimés en France tandis que le Traité de Paris l'année suivante les condamne à l'extinction au Canada. C'est alors que le Petit Séminaire doit s'improviser collège classique.

Tout ou presque tout restait à créer dans le domaine des études, a écrit un historien, car n'oublions pas que les séminaristes du régime français suivaient les cours du Collège des Jésuites. Il faudra donc désormais créer dans le Séminaire même tout un cadre d'enseignement, former un personnel de professeurs : en somme à cause de la fermeture du Collège des Jésuites, recréer tout un collège à l'intérieur du Séminaire. ${ }^{1}$

En 1765 on ouvre les premières classes. Quels furent les programmes d'études du nouveau collège ? On a dit et répété que le Séminaire a continué le système des Jésuites. Oui, si on entend le système au sens large c'est-à-dire les humanités. En fait nous savons bien peu de choses sur l'enseignement à ses débuts. Toutefois le plan d'éducation de 1790 nous apporte un renseignement fort précieux. ${ }^{2}$ Ce document nous apprend qu'on

* Thèse de licence ès lettres (Université Laval, Québec).

1 Marcel Trudel, L'Eglise canadienne sous le régime militaire (2 vol., Montréal et Québec, 1956, 1957), II : 96.

2 \& Plan d'éducation du Séminaire de Québec, octobre 1790 », documents CXV, dans Revue de l'Université Laval (RUL), XIII (nov. 1958): 270-274. Le Séminaire suit encore Batteux en 1833. Voir Exercices philosophiques littéraires... 1833. 
suit au Séminaire à l'époque «les préceptes de Mrs Rollin et Batteux ». Quand on sait l'importance qu'attache Rollin à l'étude de l'histoire et de la géographie cette remarque prend un intérêt considérable. De plus Rollin, c'est-à-dire la pédagogie de l'Université au Petit Séminaire, marquait une orientation différente de celle du Collège des Jésuites.

Il faut se garder d'exagérer ce dernier point. Comme l'a fait remarquer spirituellement Michel Bréard, « une fois délivré des Jésuites, l'Université s'installa dans leurs maisons et continua leur enseignement $\gg^{3}$ Il serait plus juste de penser que le Petit Séminaire, soucieux de mettre ses méthodes au pas des derniers progrès pédagogiques, a tout simplement suivi les théoriciens de l'enseignement les plus récents en l'occurrence ici l'abbé Batteux, disciple de Rollin.

Dans son Cours de Belles-Lettres ou Principes de littérature, édité d'abord à Paris en 1765 (5 volumes), l'abbé Batteux se contente de reprendre avec plus de précision les idées de Rollin. ${ }^{4}$ Aussi c'est la pensée pédagogique de ce dernier qu'il convient d'étudier lorsqu'on veut se faire une idée de l'enseignement de l'époque. Étude d'autant plus importante que Rollin insiste beaucoup sur l'enseignement historique et géographique et qu'il restera sur ce point le maître jusqu'au milieu du XIX Xiècle $^{\mathbf{e}}$ et même au-delà. " D'ailleurs Charles Rollin (1661-1741) n'était pas le premier venu dans l'enseignement. «Professeur, principal au collège de Beauvais, et même recteur magnifique, il a du

3 Cité par Georges Weill, Histoire de l'enseignement secondaire en France 1802-1.920 (Paris, 1920), 13.

4 La bibliothèque de l'Université a conservé 3 éditions des Principes de littérature dont la première date de 1774 et qui ont appartenu au Petit Séminaire.

5 « On n'a pas fait un pas [en éducation] depuis Rollin» (Villemain). « Dans les choses de l'éducation, le traité des Etudes est le livre unique, c'est le livre » (Nisard), cité par l'abbé Augustin Sicard, Les études classiques avant la Révolution (Paris, 1887), 42, note 1. Au Canada la vogue de Rollin est considérable. Des éditions de cet auteur sont en vente dans les librairies tout au cours du siècle. Encore en 1867 les autorités du Séminaire décident d'acheter un Rollin pour la bibliothèque des professeurs. Il semble également qu'on donne souvent des cuvres de Rollin en prix aux élèves. Un éditorial de l'Abeille du 15 février 1849 , intitulé c Lisons donc Rollin », recommande la lecture de cet auteur. 
métier », lui concède Paul Hazard ${ }^{6}$ qui par contre juge sévèrement ses idées traditionnelles. Contraint à la retraite à cause de ses idées jansénistes, Rollin composa de 1726 à 1731 son Traité des études ou De la manière d'enseigner et d'étudier les Belles-Lettres par rapport à l'esprit et au cour. ${ }^{7}$ A la vérité Rollin innove peu sauf qu'il préconise l'usage du français pour les humanités. Mais ce sont les idées de Rollin sur l'enseignement de l'histoire et de la géographie qui nous intéressent, non seulement à cause de l'influence incalculable qu'elles ont eue et que nous avons déjà signalée plus haut, mais aussi du fait de leur actualité encore aujourd'hui. Tout d'abord, Rollin s'affiche résolument disciple de Bossuet dont le Discours sur l'histoire universelle lui paraît rempli de « réflexions admirables 》 et ne peut être « trop lu ni trop estimé $\$^{8}$ Plus loin il revient sur cette idée:

Je ne puis donc trop exhorter ceux qui sont chargés de l'éducation de la Jeunesse à lire et à étudier avec attention cet excellent livre, si capable de former en même temps et l'esprit et le coeur; et après l'avoir bien étudié eux-mêmes, à tâcher d'en inspirer le goût à leurs élèves. ${ }^{9}$

Passant aux conseils pratiques, l'auteur du Traité des Études insiste d'abord sur le fait qu'il faut apporter beaucoup d'ordre

${ }^{6}$ La pensée européenne au XVIIIe siècle de Montesquieu à Lessing (3 vol., Paris, 1946), I: 259. Nous ne connaissons que par oui-dire ce qui semble être la meilleure étude sur les idées pédagogiques de Rollin, étude publiée à New-York en 1939: A. C. Gaudin, The educational views of Charles Rollin.

7 La bibliothèque de l'Université Laval possède 11 éditions du Traité des Etudes, ce qui atteste la vogue de l'œuvre. On y trouve également 14 éditions de l'Histoire ancienne et 7 éditions de l'Histoire romaine dont nous parlerons plus loin. Ajoutons à cela plusieurs atlas correspondant à ces traités et plusieurs abrégés comme ceux de l'abbé Tailhié (Abrégé d'histoire ancienne, Lyon, 1854, 1856, 1859, 1860). La grande majorité de ces ouvrages viennent de l'ancienne bibliothèque du Petit Séminaire comme en témoignent les ex-libris.

8 Charles Rollin, Traité des Etudes ou de la manière d'enseigner et d'étudier les Belles-Lettres (Paris, 1845), 64. Nous citons ici l'édition Chamerot de 1845 qui reproduit fidèlement le texte de la dernière édition publiée du vivant de l'auteur. Incidemment, l'exemplaire consulté fut donné en prix au Petit Séminaire en 1850.

9 Ibid., 456. On ne peut que difficilement aujourd'hui se faire une idée du crédit du Discours à cette époque. Voltaire, le plus grand historien du siècle des Lumières prendra la peine d'écrire l'Essai sur les Mours pour contredire le Discours. 
dans l'étude de l'histoire. C'est précisément à cette fin, enchaîne$t$-il, que contribuent la chronologie et la géographie «qu'on a raison d'appeler les deux yeux de l'histoire $\gg .^{10}$ La division de l'histoire en grandes périodes en facilite également l'étude. Ensuite il ne faut pas manquer d'observer ce qui regarde les usages et les coutumes des peuples :

comme les différentes manières de vivre, de bâtir, de faire la guerre, de former ou de soutenir des sièges, de construire des vaisseaux, de naviguer; les cérémonies pour le mariage, les funérailles, pour les sacrifices ... ${ }^{11}$

Mais tout cela, déclare Rollin, n'est encore que le squelette de l'histoire. Il faut chercher en plus la vérité, «la qualité la plus essentielle de l'historien ${ }^{12}$ et il appuie ses dires sur le témoignage d'auteurs anciens tels Cicéron, Pline et Tacite. Ensuite il cite longuement Polybe, Denys d'Halicarnasse et Bossuet pour démontrer qu'une étude profitable de l'histoire est celle qui s'applique à découvrir les causes. Rollin apparaît bien comme le disciple des historiens antiques quand il suggère à l'instar de ceux-ci qu'on étudie « le caractère des peuples et des grands hommes dont parle l'histoire ${ }^{13}$ Et comme Plutarque, il insiste sur l'étude non seulement de leur vie publique mais aussi de leur vie quotidienne privée, souvent plus révélatrice encore de leur caractère.

Passant aux recommandations plus importantes, l'auteur du Traité des Études demande de faire «observer dans l'histoire ce qui regarde les mœurs et la conduite de la vie ». ${ }^{14}$ Sur ce point encore il suit les Anciens et en particulier Tite-Live qui avait écrit dans la préface de son œuvre:

Vous y trouverez des modèles à suivre, tant pour votre conduite particulière que pour l'administration des affaires publiques; vous y trouverez aussi des actions vicieuses dans le projet, funestes pour le

10 Ibid., 424s.

11 Ibid., 451.

12 Ibid., 452.

13 Ibid., 456.

14 Ibid., 457. 
succès, qui avertissent d'éviter d'en faire des semblables. ${ }^{15}$

Enfin, dernier conseil et non le moindre: remarquer avec soin tout ce qui a rapport à la religion car, à travers toutes les superstitions grossières, « on ne laisse d'entrevoir des traces précieuses de presque toutes les vérités fondamentales de notre religion. $\gg^{16}$

Le Traité des Études est rempli d'autres conseils. Par exemple, l'auteur avertit de proportionner l'enseignement au niveau des enfants «en ne leur proposant jamais des réflexions qui soient au-dessus de leurs forces $\gg^{17} \mathrm{Et}$ ailleurs il a des pages fort bien pensées sur le déplorable fossé qui sépare à son époque l'érudition des belles-lettres. A ce sujet il conseille fortement aux maîtres de garder le goût de la science et de l'érudition traditionnelle à l'Université. ${ }^{18}$ Mais tous ces conseils s'appliquent à l'histoire de l'Antiquité et en particulier à l'histoire de Rome, « la plus riche de toutes les histoires en grands événements et en grands exemples ».19 On a reproché non sans raison à Rollin d'avoir ainsi négligé l'histoire de France que l'on enseignait déjà parfois à l'époque. Il a expliqué son attitude en invoquant le manque de temps durant les études, mais il conseille fortement aux élèves d'étudier par eux-mêmes l'histoire nationale «qui doit les intéresser davantage que celle des Grecs et des Romains et qu'il est honteux à tout bon Français d'ignorer $\gg .^{20}$

Quant à la géographie, l'auteur du Traité des Études déclare clairement qu'elle est «d'une nécessité absolue pour les jeunes gens $\gg .{ }^{21}$ L'enseignement se fera au moyen de cartes sur lesquelles on pourra même organiser des voyages imaginaires en sorte que la géographie deviendra «un divertissement plutôt qu'une étude $\gg .^{22}$

15 Loc. cit.

16 Ibid., 458.

17 Loc. cit.

18 Ibid., 684, aussi $606 \mathrm{~s}$.

19 lbid., 64.

20 Ibid,, 64. Voir aussi Sicard, op. cit., 190s.

21 Rollin, op. cit., 451.

22 Ibid., 45. 
Enfin, Rollin donne un conseil qui vaut d'être cité in extenso:

On se trouve aussi fort bien, pour leur apprendre la géographie moderne, de les engager quelquefois en famille à lire quelques pages de la gazette et de les obliger à montrer sur la carte les différents lieux dont il est parlé. Tout cela n'est point une étude: et cependant cela leur apprend la géographie d'une manière plus durable que toutes les leçons réglées qu'on leur en donne dans les formes. ${ }^{23}$

C'est pour faire suite à ses conseils sur l'enseignement de l'histoire que Rollin composa lui-même son Histoire ancienne publiée de 1730 à 1737 et son Histoire romaine, de 1738 à 1740. "Comme historien, il est sans valeur », déclare Fueter, le grand spécialiste de l'historiographie moderne. Par contre le même critique reconnaît que son immense succès «tient à ce que personne n'a su plus habilement que lui accommoder l'histoire ancienne à des vues pédagogiques et morales $»^{24}$ En somme, vers 1790, au Séminaire, on connaît Rollin et ses méthodes pédagogiques qui guideront les professeurs durant une bonne partie du XIX' siècle. Mais des traités de pédagogie aux réalisations il y a parfois loin. Aussi faut-il examiner maintenant les faits d'enseignement.

Le programme du Petit Séminaire durant cette période voit progressivement l'introduction de l'enseignement de l'histoire et de la géographie et son extension jusqu'à la Première pour l'histoire et jusqu'à la Troisième pour la géographie. C'est d'abord la géographie qui apparaît au programme d'étude en $1790^{25}$ tandis que la première mention d'un enseignement distinct de l'histoire (ancienne) remonte à 1800. ${ }^{26}$ En 1804 le cours de $359 \mathrm{~s}$.

23 Ibid., 708.

25 Plan d'éducation [...1790] déjà cité. En 1789 le mémoire célèbre de Mgr Hubert sur l'état de l'enseignement dans la colonie mentionne qu'on commence à enseigner la géographie au collège de Montréal mais ne dit rien de tel au sujet de Québec, Mandements des évêques de Québec, II : 390 s.

${ }_{26}$ ASQ, M 978 (Calendrier romain 1800; Egypte 1800, 26 pages en tout). Au collège de Montréal l'étude de l'histoire apparaît vers la même époque soit en 1798. Voir l'abbé Olivier Maurault, Le Petit Séminaire de Montréal, 85. 
géographie semble organisé pour de bon dans les quatre premières classes. Nous en étudierons plus loin le contenu. L'histoire d'Angleterre et celle de France s'enseignent au moins depuis 1808 et 1809 avec la Mythologie. ${ }^{27}$ Le programme de 1816 prescrit de la façon suivante l'enseignement de l'histoire et de la géographie : ${ }^{28}$

Septième Mythologie. Premiers principes de la géographie de l'Amérique.

Sixième Histoire Sainte. Géographie sacrée. ${ }^{29}$

Cinquième Histoire Romaine. ${ }^{30}$ Géographie ancienne. ${ }^{31}$

Quatrième Histoire Ancienne. ${ }^{32}$ Géographie moderne. Géographie de l'Europe (en partie).

Troisième Histoire de France. Géographie de l'Europe (suite et fin).

Seconde Histoire de l'Angleterre. Géographie de l'Asie et de l'Afrique.

Signalons en passant un projet intéressant qui vit le jour à l'époque. Monseigneur Plessis traça lui-même alors le plan d'étude pour le collège de Saint-Roch, succursale du Petit Séminaire. Ce programme nous intéresse en ce qu'il tendait à abréger la longueur du cours ordinaire tout en donnant aux élèves « des connaissances fort étendues dans la géographie et l'histoire ».

27 La Gazette de Québec, 17 août 1809 et 23 août 1810 . Remarquons que l'enseignement de l'histoire de France a été remis à l'honneur en France en 1806. Dès l'année suivante De Bonald signale l'apparition d'un grand nombre d'abrégés ou de manuels. Voir Marcel Reinhard, Histoire des Histoires de France dans Histoire de France [...] (2 vol., Paris, 1954), I: 17 .

28 « Plan d'éducation du Séminaire de Québec 1816 », documents CXXVI, dans RUL, XIII (janvier 1959) : 463s. sainte.

29 La géographie du monde biblique pour aider à l'étude de l'histoire plus bas.

${ }^{30}$ Qui se termine alors à la naissance du Christ comme nous verrons

31 Géographie du monde antique tel que les Anciens se le représentaient et utile pour l'étude des textes grecs et latins.

32 Histoire des peuples de l'Antiquité à l'exclusion des Romains. Sens courant à l'époque que nous avons rencontré chez Rollin. 
Mais le collège connut une existence trop éphémère pour permettre cette expérience pédagogique nouvelle. ${ }^{33}$

Les professeurs de l'époque ne semblent ni par leur formation ni leurs goûts s'intéresser particulièrement à l'histoire ou

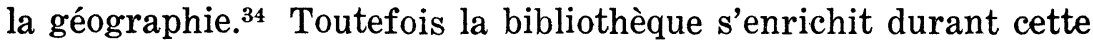
période de nombreux ouvrages relatifs à ces deux disciplines. D'abord la bibliothèque du Séminaire hérite de nombreux ouvrages de celle, dispersée, du collège de Québec comme en font foi les ex-libris de ces volumes aujourd'hui à la Bibliothèque de l'Université Laval. Plusieurs prêtres, comme le curé Récher, les abbés Leguerne et Parant, Mgr Briand, et plus tard Mgr Plessis, lèguent au Séminaire leurs bibliothèques personnelles souvent très riches d'ouvrages historiques et géographiques. Enfin, il semble qu'on importe beaucoup de livres de France entre 1765 et $1830 .{ }^{35}$

Nous avons la bonne fortune de posséder un catalogue de la Bibliothèque du Petit Séminaire en 1782 qui renseigne sur les lectures historiques et géographiques du temps. ${ }^{36}$ Sur les 5,024 volumes recensés, 520 traitent d'histoire, auxquels il faut ajouter plusieurs ouvrages d'histoire et de géographie rangés dans la catégorie Belles-Lettres, riche de 577 volumes. On trouve dans

33 L'abbé Jean-Baptiste Ferland, Mgr Joseph-Octave Plessis, évêque de Québec (Québec, 1876), 147 ss. Voir aussi « Le Collège classique de Saint-Roch de Québec », par l'abbé Ivanhoë Caron dans BRH, XLV (1939): 97-100.

34 Comme l'a déjà fait remarquer l'abbé Arthur Maheux dans « Jacques Labrie 》, MSRC, XLII (1948) : 124.

35 Nous n'avons pas l'intention de rouvrir ici le débat sur la venue des livres français avant 1850. Il semble que malgré les frais élevés de la douane et les prix souvent presque prohibitifs des ouvrages, le commerce n'ait jamais été interrompu durant ce temps. Benjamin Sulte a écrit il y a longtemps à ce sujet (« Nos Ancêtres étaient-ils ignorants ? dans MSRC, XII (1918): 203): \& je vois par les arrivages des navires d'Angleterre, dans les vieilles gazettes, que les chargements comprenaient des caisses de livres français adressés à nos marchands et cela explique les éditions de $1770,1775,1790$ trouvées dans maintes et maintes bibliothèques. 》 Voir également M. Marcel Trudel, «A propos de cette unique grammaire de $1760 \gg$, dans RUL, IX (1954-55): 780-5.

36 A. G. Dudevant, ptre, Catalogue des livres de la bibliothèque du Séminaire des Missions Etrangères de Québec fait dans le mois de May 1782. Sur l'auteur et le catalogue voir l'article de $M$. Antonio Drolet: * La Bibliothèque du Séminaire de Québec et son catalogue de $1782 \%$, dans le Canada français, XXVIII (1940) : 261-266. 
la bibliothèque des écrits de tous genres: traités, manuels, abrégés, dictionnaires. Pour n'en citer que quelques-uns:

L'Histoire du Canada de Charlevoix (4 volumes)

$L ' H i s t o i r e$ ancienne de Rollin (13 volumes)

L'Histoire de France de Mézeray (3 volumes)

5 volumes d'Oeuvres de Voltaire

L'Histoire universelle de Bossuet (2 volumes)

La Géographie sacrée de Mr Robert (2 volumes)

La Géographie universelle du père Buffier

La Géographie moderne de M. Lacroix (2 volumes)

Les Cérémonies de la Chine par le père Lombardie

La bibliothèque ne compte que 5 ouvrages de Voltaire et encore sont-ils dépareillés, faisant originairement partie d'une édition des œuvres du philosophe en 12 volumes. Si on a pu dire que pendant toute la période qui nous intéresse ici, Voltaire a été notre "seul maître d'histoire », ${ }^{37}$ il faut faire observer que c'est un Voltaire connu hors des collèges par des extraits d'articles de journaux ou des volumes empruntés dans des bibliothèques comme la Bibliothèque publique de Québec. Un ancien élève du Séminaire, devenu plus tard le célèbre Papineau, s'est dit-on vanté un jour d'avoir perdu la foi au Petit Séminaire à l'occasion de lectures impies. Lectures faites certes en dehors de la bibliothèque de l'institution. D'ailleurs nous savons que Papineau avait accès à la Bibliothèque publique de Québec, très libérale, semble-t-il, dans ses prêts. ${ }^{38}$

Après 1792 la bibliothèque du Séminaire continue de s'enrichir de volumes d'histoire. Les rééditions de Rollin, celles d'Anquetil (depuis 1805) et de Mézeray s'accumulent sur les rayons avec régularité. Un exemple de cette régularité nous

${ }^{37}$ Marcel Trudel, L'influence de Voltaire au Canada (2 vol., Montréal, 1945), II: 254.

38 L'abbé Groulx, dans une étude sur «Les idées religieuses de Papineau », publiée dans Notre Maître le passé ( $2^{\mathrm{e}}$ série), écrit (p. 184): * Papineau, après avoir épuisé la bibliothèque de ses professeurs Lahaille, Robert et Demers et celle du Séminaire, souscrit au cours de sa deuxième année de philosophie à la Bibliothèque publique de Québec. 》 
est fourni par l'acquisition entre 1815 et 1825 d'une vingtaine d'ouvrages d'une conception nouvelle et propre à intéresser les jeunes à l'histoire et à la géographie. Il s'agit de volumes de vulgarisation intitulés Beautés de ... La Bibliothèque du Séminaire possédait entre autres les Beautés de l'histoire ancienne ou faits et dits mémorables des grands hommes [. . .], les Beautés de l'histoire d'Amérique (en partie), 2 éditions des Beautés de l'histoire d'Angleterre, les Beautés de l'histoire de Chine, du Japon et des Tartares, les Beautés de l'histoire de la Grèce moderne, les Beautés de l'histoire des États-Unis d'Amérique Septentrionale, ou précis des événements les plus remarquables concernant ces différents États [...] etc. Ce genre semble avoir eu son origine en Angleterre. Nous avons retrouvé un volume intitulé The Beauties of Shakespeare, publié à Londres en 1783. Plus que les programmes d'étude et plus que les lectures qui restent une activité marginale, le contenu des cours d'histoire nous aide à mieux connaître l'évolution de l'enseignement. De cette période, il nous reste quelques manuels français, ${ }^{39}$ mais il est difficile de préciser jusqu'à quel point ils ont pu servir. Par contre nous possédons une source plus intéressante dans quelques rares cahiers de notes de cours prises par les élèves euxmêmes. Bien entendu, conformément aux conseils de Rollin et à l'esprit de l'époque, l'histoire ancienne opposée à l'histoire moderne a la part du lion..$^{40}$ Et le cours se termine habituellement de la façon suivante:

quelques années après la bataille d'Actium, Octavien prit le nom d'Auguste et le titre d'empereur; tout céda à sa fortune [...], il ferma le temple de Janus et donna la paix à tout l'univers. C'est la 42ème

39 Depuis l'Histoire de France et l'histoire romaine par demandes \& par Réponses [...] (2 volumes totalisant plus de 1200 pages; ex-libris de Récher, 1757, puis celui du Séminaire, 1782) jusqu'aux Tableaux chronologiques de l'histoire ancienne et moderne tant sacrée que profane [...] (une édition de Paris en 1824, une autre de Lyon en 1829).

40 L'histoire * considérée du côté du temps [...] se divise en ancienne qui s'étend jusqu'à la naissance du [Christ] et en moderne qui renferme les événements arrivées (sic) depuis [...] \$, ASQ M-61. Le cahier M-978 déjà signalé, renferme sous le titre Egypte un petit cours d'histoire ancienne où l'on étudie Egyptiens, Phéniciens, Assyriens et Babyloniens, Perses et Mèdes et enfin les Grecs dont l'histoire occupe 17 des 22 pages de l'exposé. 
année de son règne, l'an 4004 du monde que naquit Jésus-Christ..1

Les seules sources dont nous disposons pour l'étude de l'histoire moderne à cette époque consistent dans deux cahiers reproduisant le même cours d'histoire de l'Angleterre donné en 1828.42 Il s'agit d'une histoire divisée par règnes et rapportant presque exclusivement les faits et gestes des monarques. Très flatteuse pour le souverain régnant elle se termine par une apothéose de l'Angleterre après Aboukir.43 L'hospitalité anglaise, offerte après 1789 «aux misérables citoyens persécutés par les Tirans (sic) les plus inhumains », est célébrée comme « un monument éternel de l'humanité et de la générosité du gouvernement britannique $»^{44}$ Et le professeur stigmatise cette

révolution générale qui a étonné le monde entier, qui a menacé, troublé la tranquilité de l'Europe, révolution parricide qui a décapité le plus ignomineusement, pour des crimes supposés, le plus religieux et le plus paisible des souverains: révolution sanguinaire qui a commôncé (sic) par le feu, continué par le massacre et inventé pour accélérer, un nouvel instrument de supplice. ${ }^{45}$

Apothéose de l'Angleterre, dénigrement de la France de 89 et, comme corollaire attendu, célébration des bienfaits du régime anglais au Canada:

Enfin, la conquête de cette immense Province fut terminée par une haute capitulation très favorable aux François [...]. Depuis ce temps-là le peuple canadien a le bonheur inestimable de vivre sous la douce influence du gouvernement Britannique. ${ }^{48}$

Ces cours se donnent en 1828, au moment de la lutte aigre au sujet des subsides, moins de dix ans avant l'explosion na-

41 ASQ, M-91, 67. Cette phrase se retrouve mot à mot dans L'abrégé d'Histoire ancienne et sacrée, publié à Montréal en 1835 (p. 101).

12 ASQ M-55 et M-56.

43 ASQ M-55, 108.

14 Ibid., 100.

46 Ibid., 45.

16 Ibid., 93s. 
tionaliste de 1837. Un cahier de notes d'élève prises à l'occasion d'un cours de belles-lettres nous renseigne sur la conception de l'histoire et du métier d'historien à l'époque. ${ }^{47}$ Le professeur démarque le Projet d'un traité sur l'histoire extrait de la Lettre à l'Académie. Suivant Fénelon, il écarte de l'histoire les discours fictifs chers à l'Antiquité tels que les avait pratiqués Thucydide. De plus, non seulement l'historien n'a pas de «passion », mais encore il n'a «ni amis, ni ennemis, ni parents, ni patrie ». Car «il n'a rien à prouver ni à détruire, il n'excuse ni ne défend». En somme, "tout son office est d'exposer la chose comme elle est. » Et le cours continue sur ce ton. Le Projet d'un traité sur l'histoire reste encore aujourd'hui au programme de littérature ${ }^{48}$ et constitue à peu près la seule initiation théorique à la méthode historique au cours secondaire. Signalons enfin que l'étude de l'histoire est encouragée à cette époque par la distribution d'ouvrages historiques comme prix, tel ce manuel d'histoire grecque donné en 1811.49 Toutefois les premiers prix d'histoire n'apparaîtront qu'en 1833 et leur attribution fera l'objet d'un concours spécial.50

Nous avons déjà vu que l'enseignement de la géographie pénètre avant celui de l'histoire au Séminaire. En fait il s'agit d'un enseignement bien sommaire. Un cahier de notes de géographie de 1790 renferme une copie d'extraits de Buffier que l'on utilisait alors comme ouvrage de base. L'étude de notre continent, région bien exotique pour le petit Français du temps, est réduite à sa plus simple expression et présentée en vers pour faciliter le travail de mémorisation :

47 ASQ M-95 (Cours abrégé des belles-lettres). Sur l'histoire, 127-130.

48 Université Laval, Faculté des Arts, "Cours classiques de garçons. Règlement de l'Immatriculation et du Baccalauréat 》, Programmes des Etudes, No 20, 73.

49 Abrégé de l'histoire ancienne en particulier de l'histoire grecque, suivi d'un abrégé de la fable, à l'usage des élèves de l'école royale militaire de Paris [...]. Faisant partie du cours d'études rédigé \& imprimé originairement en France. Par ordre de Louis XVI (Londres, 1808). Conservé à la bibliothèque de l'Université Laval.

50 Exercice philosophique [...] pour 1833. 


\section{L'AMERIQUE}

L'Amérique, Du Nord par l'isthme panama,

Se divise au Sud, Québec en Canada.

Boston avec Charlestown en Nouvelle Angleterre,

Le fort S. Augustin Dans la Floride Terre,

Santa Fé, Capitale au Mexique Nouveau;

Et la Nouvelle Espagne a pour chef Mexico.

Partie Méridionale de l'Amérique

Terre Ferme, Pérou, dans le Sud d'Amérique,

Au Chili San-jago Puis la Majellanique.

La Plata, le Brésil, qui tient San-Salvador,

Aux Amazones sont Les Villages de l'Or

Iles De l'Amérique

Açores, Terre-Neuve, Antilles, Vers Mexique, Aux Antilles Petits se voit La Martinique

Rivières de l'Amérique

Au Nord Mississipi, le Fleuve Saint-Laurent,

Au Midi L'Amazone, le Fleuve D'argent.51

Les deux-tiers du cahier de 22 pages sont consacrés à l'Europe et le tiers à la France. Il s'agit tout au long de la même énumération versifiée de régions, villes, fleuves.

On a aussi de 1801 un cahier de notes de cours intitulé Principes de Géographie. Encore ici il s'agit d'une énumération sèche qui, après quelques mots sur l'étendue et les bornes des continents, parle successivement de la «division des terres » (montagnes, caps, îles), de celle des eaux (mers, golfes, détroits, lacs et fleuves). Viennent ensuite les «divisions politiques»

51 ASQ, M-272, 20ss. 
(liste des états avec leur capitale). Enfin le tout s'achève par des «Notions générales concernant les peuples» (langues, religions, forme de gouvernement et qualité du souverain s'il y a lieu). L'Amérique, étudiée après l'Asie et l'Afrique, occupe le quart du cours. ${ }^{52}$

1804 est à marquer d'une pierre blanche dans l'histoire de l'enseignement de la géographie au Petit Séminaire. En effet, c'est cette année-là que l'on réorganise l'enseignement de cette discipline en publiant un manuel et en faisant fabriquer des cartes. Ces dernières, conçues spécialement dans un but pédagogique, concordent avec l'enseignement du manuel destiné à l'usage des écoliers du Séminaire. Au nombre de 41, cinq d'entre elles concernent l'Amérique. Sans doute les affichait-on, car elles sont trouées. ${ }^{53}$

Le manuel intitulé Géographie à l'usage des écoliers du Petit Séminaire de Québec qu'on a salué comme le premier manuel canadien de géographie, ${ }^{54}$ peut être attribué à l'abbé François Pigeon, professeur du temps. ${ }^{55}$ Dans son avertissement, l'auteur nous dit qu'il a rédigé cet opuscule pour éviter aux étudiants les inconvénients de la consultation de " gros volumes », de «l'incommodité et la peine de copier des abrégés » ainsi que dans le but d'uniformiser les programmes. De plus l'auteur «ne prétend donner qu'une idée générale de cette Science (qui n'est qu'accessoire à un cours d'Etudes ordinaires) », n'ayant voulu que «disposer les Enfants qui par goût ou par état voudront en faire une Étude particulière». Enfin, l'auteur annonce qu'il ne tiendra

52 ASQ, M-978.

53 ASQ, Tiroir 223, no 1. (Atlas) pour servir à la Géographie du Petit Séminaire de Québec ». L'une des cartes porte l'indication: "par Chs Fairbank ». Les Archives possèdent aussi une série de 12 cartes toutes éditées avant 1792 sur l'Ancien Testament, les découvertes de Cook, etc., cartes qui ont pu servir à l'enseignement de la géographie. Plusieurs portent au verso la signature de «Gravé 》, ASQ, Tiroir 223, no 2. 54 Philéas Gagnon, Essai de bibliographie canadienne (2 vol., Québec, 1895, 1913), I: 202.

55 Une main anonyme a ajouté le nom de l'abbé Pigeon sur la page titre d'un exemplaire conservé à la Bibliothèque de l'Université Laval. L'abbé est professeur au Petit Séminaire à ce moment-là. Il quittera peu après. 


\section{L'A F RI QU E.}

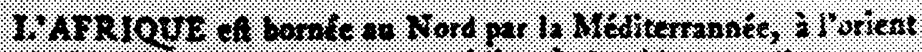

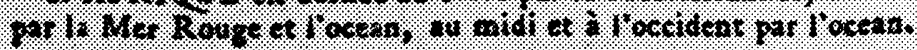

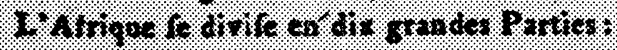

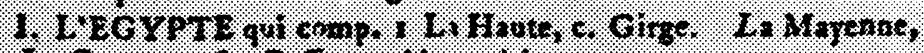

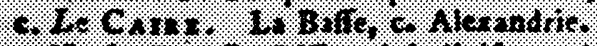

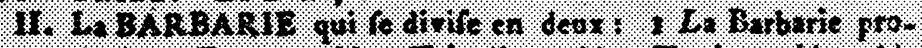

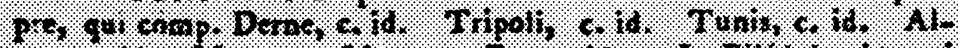

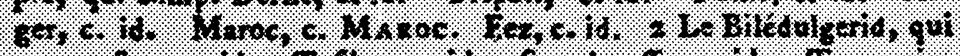

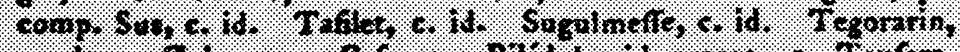

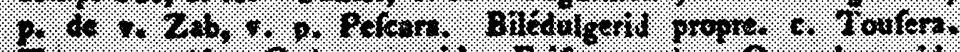

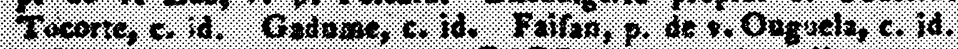

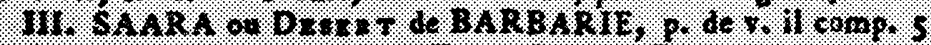

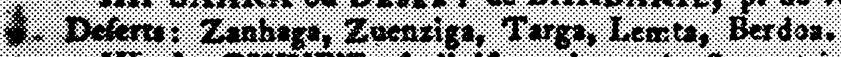

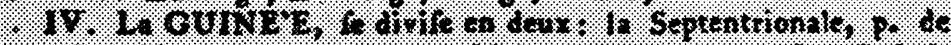

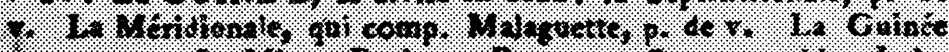
F.

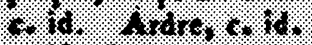

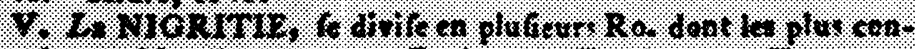

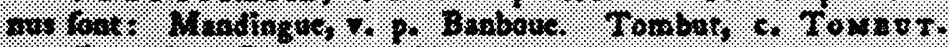

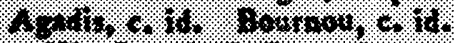

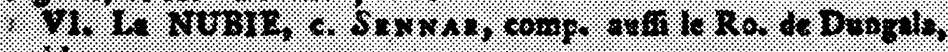
0.10

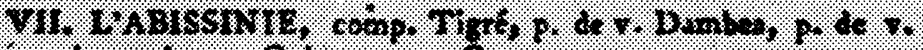

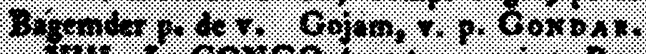

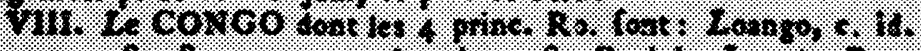

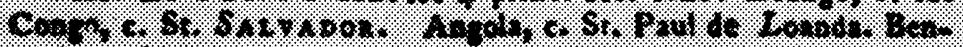
$1.0 .10 .10 .1 \%$

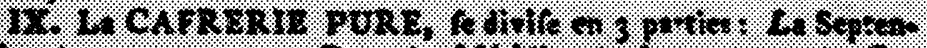

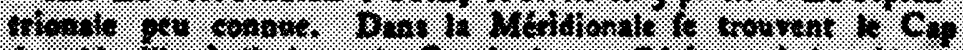

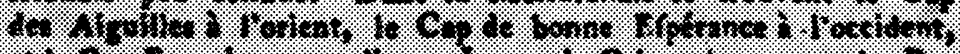

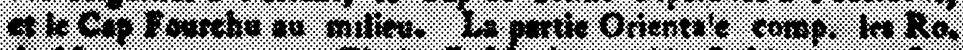

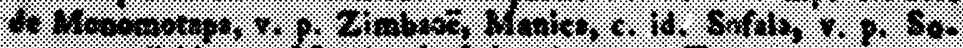

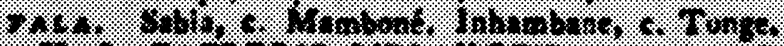

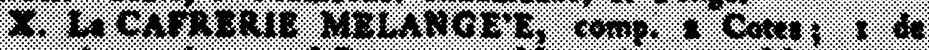

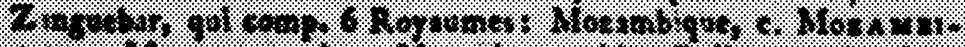

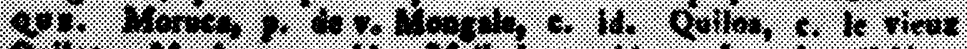

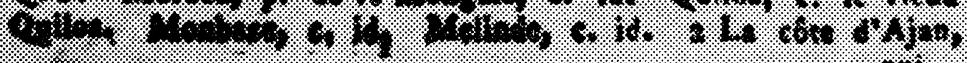
1.

Une page de la Géographie à l'usage des écoliers du Petit Séminaire de Québec, publiée en 1804 . Grandeur réelle: $10 \times 14.5 \mathrm{~cm}$. 
pas compte dans son ouvrage des bouleversements occasionnés par la Révolution française. ${ }^{56}$

Le contenu du manuel est divisé suivant les classes où l'on enseigne la géographie et constitue le programme probablement suivi jusqu'à l'abbé Holmes. Après les notions générales sur la géographie et les termes géographiques, on étudie l'Amérique comme premier continent (ce qui constitue une nouveauté), puis l'Europe, l'Asie, l'Afrique et les terres polaires. L'auteur ajoute un abrégé de géographie sacrée qui consiste en une description du pays des patriarches et de la Judée. Suit une brève histoire de la Terre-Sainte depuis le Christ jusqu'au XIX ${ }^{\mathbf{e}}$ siècle. Enfin, l'ouvrage s'achève par un abrégé de Géographie ancienne ou «manière dont les Anciens divisaient la Terre.$^{57}$ Cette étude apparaissait alors très utile pour la compréhension des textes grecs et romains. Encore là bien entendu il s'agit d'énumérations avant tout. Au chapitre Canada, par exemple, on cite d'abord les 21 comtés du Bas-Canada et les villes importantes puis s'ajoutent quelques mots seulement sur le reste du pays:

Le Haut-Canada, ville unique Kingston. Au Nord du Canada est une vaste contrée, séparée par la Baie d'Hudson. La partie orientale se nomme Labrador et les habitants Esquimaux. L'Occidentale est peu connue. Au Sud-Ouest, le Nouveau-Brunswick, c [apitale] Frédéricton. A l'Orient, l'Acadie ou N [ouvelle] Ecosse, $c$ [apitale] Halifax. ${ }^{58}$

En somme, malgré ses déficiences inhérentes à l'état de la science et de la pédagogie de l'époque, le programme de 1804, par l'introduction de cartes "pédagogiques », la graduation intelligente du programme et la place accordée à l'étude de l'Amérique, marque un progrès décisif. On a déjà à ce moment une esquisse du programme actuel de géographie, esquisse que l'abbé Holmes précisera et complétera un peu plus tard.

56 [L'abbé François Pigeon], Géographie à l'usage des écoliers du Petit Séminaire (Québec, 1804). Avertissement. La carte de la France correspondant au manuel divise encore le pays en 32 gouvernements connus sous l'Ancien Régime.

57 Ibid., 24.

58 Ibid., 4. 
A cette époque on distribue également en prix des volumes de géographie comme cette Géographie moderne de Nicolle de la Croix donnée en 1808 à Charles Turgeon, élève de Troisième. ${ }^{59}$

\section{(à suivre)}

Pierre SAVARD, assistant à la Faculté des Arts (Laval).

59 Conservée à la bibliothèque de l'Université Laval. 